\title{
ARE LOW BIRTH WEIGHT INFANTS AT RISK FOR MINERAL DISTURBANCE IN EARLY NEONATAL PERIOD?
}

\author{
L.D. Blaga, G. Zaharie, M. Matyas, M. Popa \\ Neonatology, University of Medecine and Pharmacy, Cluj-Napoca, Romania
}

Bacground and aims: The physiological levels of calcium and magnesium are very unstable in the first few days after birth, due to abrupt interruption of placental supply, low level of PTH and high levels of calcitonin, physiological acidosis. Infants with intrauterine growth retardation and preterm birth are at risk for hypocalcemia or hypomagnesemia. Often asymptomatic they can be undiscovered and untreated. There are a lot of controversies in regards with the physiological low limit of calcium and magnesium; are their values, smaller than those which are biostatistic classified, without any clinical and electrocardiographic aspects, physiological or not for ontogenetic age?

The aim of these paper is to establish the incidence of early hypocalcemia and hypomagnesemia in low birth weight infants and the risk factors that impose the cases in a screening program.

Methods: Calcium and magnesium were determined in 120 newborns (50 term SGA newborns, 50 prematures and 20 AGA newborns), 48 hours after birth.

Results: Hypocalcemia was detected in $16 \%$ of term SGA with Rohrer index below 1.8 and $16 \%$ of prematures with gestational age under 32 weeks and birth weight below 1500g. Hypomagnesemia was detected in $4 \%$ of term SGA and $16 \%$ of SGA prematures. 4 term SGA have had hypocalcemia and hypomagnesemia. Onely 5 cases of term SGA with hypocalcemia were simptomatic.

Conclusions: Low birth weight infants and severe intrauterine growth retardation are high risk factors for mineral disturbance. Screening is usefull in SGA new bornes with low Rohrer index and prematures under $1500 \mathrm{~g}$. 